\title{
Technical Note: Positron Emission Tomography- Computed Tomography (PETCT) Guided Lung Biopsy for Better Yield - A Single Center Experience
}

\author{
Shiva kumar Swamy Shivalingappa, Mahesh Ashok Kumar, Kumar Kallur, Indiresh Desai, G Lohith*, \\ Sudhakar S, Prashanth G R, Avinash Kesari, Raj kumar and Basavalinga Ajai Kumar
}

Consultant Radiation Oncologist, Healthcare global enterprises Limited, India

Received: August 29, 2017; Published: September 08, 2017

*Corresponding author: G Lohith, Consultant Radiation Oncologist, Healthcare Global Enterprises Limited, India, Tel: 919620 251171;

Email: lampard_buddyy@hotmail.com

\section{Introduction}

Tissue diagnosis is mandatory in work up and treatment strategies of lung cancers. There are many techniques in obtaining tumor samples which include less invasive and invasive techniques. Image guided percutaneous biopsy plays an important role in the work up for lung cancers, of which percutaneous computed tomography (CT) guided core biopsy is an important component. Recent advances in target therapies and personalized medicine, the technique of aspiration cytology is not a preferred technique. The need for multiple large cores of tissue for the diagnoses which include histopathology, immuno histochemistry and also formulation studies is the need of hour and CT guided technique is preferred. The procedure is less invasive, often performed under local anesthesia and as a day care procedure. Complications of CT guided lung biopsy have been well documented and include pneumothorax, haemoptysis, pain, air embolism, a trial fibrillation, and tumor seeding of the biopsy tract and, on rare occasions, death. The complication rates vary from center to center, owing to the expertise. It is important for the interventional radiologist to perform the procedure in one go. Even with best of the best practices, improvised techniques and technique modifications in minimizing complications, we quite often we face a situation of inadequate tissue sample. This leads to repeat procedures, cost and exposes the patient for procedure related complications. All these lead to delay in diagnosis and initiation of treatment. A well performed diagnostic CT scan, preferably with intravenous contrast is crucial in planning the procedure. The incidences of repeat procedures are high with necrotic tumors, central hilar masses and when there is presence of adjacent atelectasis.

\section{Material and Methods}

20 elderly individuals diagnosed to have pulmonary mass on CT scan images were considered for PETCT studies. We selected central lung masses which were masqueraded by the atelectasis lung. These patients underwent PETCT guided percutaneous biopsy procedures. The biopsy samples were obtained from the most PET avid areas. 18G coaxial (BARD automatic max core and semiautomatic truglide coaxial disposable biopsy instrument) system was used for the procedure. Biopsy throw of $2.2 \mathrm{~cm}$ and minimum of 5 and maximum of 10 cores were obtained.

\section{Results}

All cores were further analyzed by an oncopathologist. All sampled cores defined cancer cells and were adequate for various other tests.

\section{Conclusion}

Guided core biopsy plays an important role in obtaining tissue samples for analysis. CT guidance is routinely used for lung biopsies. Quite often the sample biopsied may not be true representative of tumor cells. The use of PETCT guidance in those instances helps in tissue sampling, thereby minimizing the repeat procedures and also significantly reduces patient morbidity. In our series of 20 patients, all the cores showed cancer cells and was sufficient for various other tissue analysis and hence we recommend PETCT guided biopsies. 


\begin{tabular}{|l} 
BIOMEDICAL \\
RESEARCHES
\end{tabular}$\quad \begin{aligned} & \text { Assets of Publishing with us } \\
& \text { - Immediate, unrestricted online access }\end{aligned}$

